\title{
In a forest of airway guidelines, it's time to make a shared path
}

\author{
Laura V. Duggan, MD, FRCPC $\mathbb{D}$
}

Received: 18 May 2021/Revised: 18 May 2021 / Accepted: 25 May 2021/Published online: 6 July 2021

(C) Canadian Anesthesiologists' Society 2021

The medical literature has undergone exponential growth over the last 60 years, and anesthesiology is no exception. ${ }^{1}$ No one person could stay current-let alone integrate relevant primary literature into practice-hence the growth of synthesis literature in the form of various types of reviews and clinical practice guidelines. A sizable body of scholarly work has been published on how to create clinical practice guidelines $(\mathrm{CPGs}){ }^{2}$ Knowledge translation ${ }^{3}-$ in the form of effective dissemination, measured adoption and practice change, and, most importantly, quantification of improvement in patient-centred outcomes-often remains unclear.

Clinical practice guidelines are published by various groups and societies. Meant to assist in clinical decisionmaking, CPGs can be at odds with one another depending on the evidence used, processes, and opinions of its members. Clinical practice guidelines should include an iterative plan once published - or risk becoming quickly irrelevant. The first difficult airway management guidelines were published in 1993 by the American Society of Anesthesiologists (ASA). ${ }^{4}$ The inaugural Canadian Airway Focus Group (CAFG) recommendations were published in 1998. ${ }^{5}$ In 2013, the CAFG recommendations were revised as two separate articles: the first addressing the unanticipated difficult airway ${ }^{6}$ and the second addressing the anticipated difficult airway. ${ }^{7}$ To date, the CAFG recommendations have been the only major airway CPGs to publish an algorithm specifically addressing the patient with an anticipated difficult airway. Overall, in a recent directed review, a total of 38 difficult airway algorithms

L. V. Duggan, MD, FRCPC $(\bowtie)$

Department of Anesthesiology and Pain Medicine, Ottawa Civic Hospital, University of Ottawa, Ottawa, ON, Canada

e-mail: laduggan@toh.ca have been identified, with $58 \%$ published in the last five years (Figure). ${ }^{8}$

In this edition of the Journal, Howard et al. ${ }^{9}$ report on the findings of a survey on the use of difficult airway guidelines in anesthesiology practice in the London, Ontario area, with specific questions relating to the awareness and use of the CAFG guidelines. Their survey included consultant anesthesiologists and trainees from two academic centres; a separate survey on departmental adoption of guidelines was sent to the department heads of 21 surrounding community anesthesia departments. The survey asked respondents which difficult airway guideline they followed (if any), if their department endorsed a specific difficult airway guideline, and about awareness of the CAFG guidelines. In addition, the survey explored respondents' personal comfort level with technical skills from commonly used techniques such as videolaryngoscopy to infrequent, yet core competency skills involved in establishing emergency front-of-neck airway (eFONA), usually during a "cannot ventilate, cannot oxygenate" (CVCO) emergency.

Survey response rates in the two academic centres were reasonable: $74 \%(28 / 38)$ of trainees and 68\% (51/74) of academic consultants took part. Nevertheless, the response from regional department heads was somewhat disappointing, with only $52 \% \quad(11 / 21)$ choosing to participate; therefore, patterns or conclusions gleaned from the regional anesthesia departments are limited.

A notable minority of $25 \%$ of academic consultants (13/ $51)$ and a small number of trainees (7\% [2/28]) responded that they did not follow any published airway guideline. Of the consultants not using a CPG, the most common reason cited was the view that airway management should not be standardized. There is merit in this opinion; every patient deserves an airway strategy (i.e., a coordinated series of 
Figure Number of difficult airway management algorithm publications from 1998 to 2018 (reproduced with permission from Edelman et al.). ${ }^{8}$ The blue bars show the number of publications per year; the orange bars indicate the cumulative number of algorithms published. Nombre de publications d'algorithmes de prise en charge des voies aériennes difficiles de 1998 à 2018 (reproduit avec la permission d'Edelman et coll. $)^{8}$ Les barres bleues indiquent le nombre de publications par année; les barres orange indiquent le nombre cumulé d'algorithmes publiés.

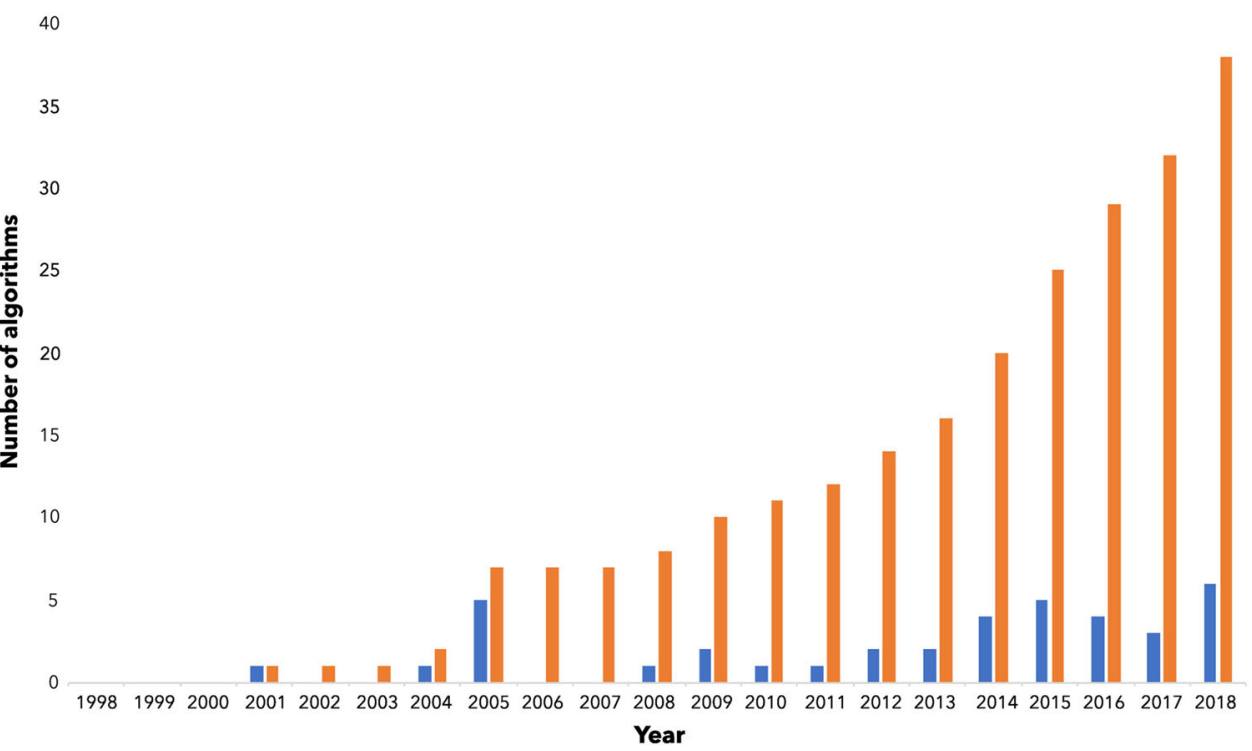

plans) based on their individual characteristics and circumstances. The irony is that airway guidelines do not espouse "one airway strategy for all" but rather highlight important factors (e.g., personnel and equipment availability, patient physiology) to consider when constructing an individual airway strategy.

Three main domains comprise airway management expertise development: appropriate context-specific decision-making, technical skills, and predictable teamwork. Although a CPG can assist with decision-making, technical skills have traditionally been learned through an apprenticeship model. ${ }^{10}$ The apprenticeship model is based on the assumption that, with enough time, the learner will have adequate exposure to all aspects of airway management decision-making and technical skills through sheer patient volume. Although this may achieve appropriate training for more commonly used skills (e.g., Howard et al. found that $90 \%$ of respondents felt comfortable with videolaryngoscopy), such comfort level may not necessarily translate to decision-making during an emergency ${ }^{11}$ or to performance of rare skills (e.g., cricothyrotomy). The apprenticeship model also supports a hidden curriculum-i.e., that airway management is an individual and not a team endeavour.

Guidelines followed by respondents in Howard et al.'s survey included those from the ASA, CAFG, and the Difficult Airway Society. ${ }^{12}$ Although $72 \%$ of all respondents were aware of the CAFG guidelines, many cited issues with knowledge translation. Criticisms included the lack of society endorsement, lack of adequate dissemination, and the lack of associated cognitive aids to assist the airway manager during patient care. All these criticisms are valid. It is not adequate to publish a CPG and leave it to individual clinicians to find and translate them into action. Clinical practice guidelines have a responsibility to make themselves user-friendly, adaptable for local practice, and include cognitive aids/ triggers to encourage their use. Although society endorsement is traditional, airway management is practiced by several acute care specialties (e.g., emergency medicine, intensive care, anesthesiology) and other healthcare fields (e.g., respiratory therapy, prehospital paramedic care). It is time to create a Canadian Airway Network to connect airway leads across specialties and not just through intermittent CAFG guidelines. This network is now being developed. Departmental airway leads, a concept borne from the UK's 4th National Audit Project, ${ }^{13}$ is a means of connecting a CPG to a clear point person at a local level. Airway leads are now present and working in the UK and New Zealand to translate knowledge, ${ }^{14}$ and are a means for making CPG iterative and responsive to local requirements.

Notably, in the survey by Howard et al., $25 \%$ of trainees stated that they were taught and followed multiple guidelines. While not necessarily wrong per se, this also highlights a paradox: half of the trainees also reported inadequate training in difficult airway management. In this sense, Howard et al.'s study shows the weakness of apprenticeship: it may overteach some aspects of airway management while underteaching others. All trainees deserve a well-designed deliberate airway curriculum that is consistent, evidence-based, and complete. It needs to include the decision-making, technical, and teamwork skills required for the prevention and management of airway emergencies. Without clearly delineating, teaching, and assessing these skills, we are doing our trainees-and our patients-a disservice. 
Finally, no difficult airway CPG is complete without specifically addressing the CVCO emergency. Even though there are many difficult airway $\mathrm{CPGs}^{8}$ the $\mathrm{CVCO}$ emergency approach is fairly consistent. Performance of an eFONA is a core skill of advanced airway competency. All advanced airway managers have a responsibility to know when and how to perform an eFONA - in the hands of non-surgeons, generally in the form of a cricothyrotomy. Howard et al. found that only a minority of trainees (4-7\%) and consultants (18-24\%) felt comfortable performing either a needle- or scalpel-based cricothyrotomy. This is reflected in malpractice claims related to difficult tracheal intubation in the ASA Closed Claims Project database, where delay to eFONA, generally in the form of waiting for a surgeon, was cited as a common theme in the majority of reported cases. ${ }^{15}$ There are now easily accessible resources to practice cricothyrotomy. ${ }^{16}$ Perhaps Howard et al.'s finding that only $13 \%$ of respondents had practiced an eFONA in the last year and $43 \%$ had never practiced an eFONA can now be addressed.

How can we do better? Appropriate CVCO management should not solely depend on an individual airway manager's situational awareness, decision-making, and cognitive and technical skills being performed accurately and in a timely manner. It is unrealistic and potentially career-destroying for the airway manager, unnecessarily risky for the patient, and damaging for the team, the latter of which oftentimes contains individuals with better situational awareness than the airway manager. The difficult tracheal intubation-related ASA Closed Claims Project database analysis found perseveration of tracheal intubation attempts to be a common theme ${ }^{15}$; this challenges the term "can't intubate" especially if the airway manager has the responsibility of determining this. The Journal's recent publication on specialist anesthesiologist airway management-related closed civil legal cases from the Canadian Medical Protective Association found that the majority of patients who had suffered brain damage or death were ASA Physical Status I-III patients presenting for elective surgery. ${ }^{17}$ "Cannot ventilate, cannot oxygenate" can occur at any time; having predictable responses to airway emergencies within the confines of local equipment, skills, and practice will frequently make the difference between the life and death of a patient.

Acute trauma care is managed using a standardized Advanced Trauma Life Support (ATLS) approach such that no serious injuries are missed, and multiple healthcare providers can perform tasks simultaneously with one trauma leader. The specifics of injuries can then be addressed. Acute cardiac conditions are managed using a standardized Advanced Cardiac Life Support (ACLS) approach to restore circulation. Chest compressions are anticipated and performed by a rotating group of people while standardized drugs are given. The specifics of rhythm management can then be the focus. The leader is at the end of the bed. Without the team dividing tasks and preparing equipment for anticipated next steps, care is delayed. It is time to have a standardized approach to airway emergencies. Although there are many difficult airway CPGs, CVCO can be recognized by the team through waveform capnography and oximetric desaturation, usually in that order. This does not depend on the airway manager determining the event occurring. In an advanced airway approach, this would be immediately followed by passing the airway manager a supraglottic device while a code airway is called. An eFONA is expected if bag-mask ventilation, use of a supraglottic device, and tracheal intubation by videolaryngoscopy and direct laryngoscopy fails to oxygenate the patient. The frequency of practicing for this event can be similar to the recertification of these other emergencies. While not perfect, providing a scaffolding of standard responses frees up cognitive space to problem solve for the specific situation, much like ATLS and ACLS. Practice makes the unfamiliar familiar, and CVCO emergencies, along with eFONA performance, are no different.

In conclusion, Howard et al. have provided us with the important insight that producing a difficult airway CPG (let alone 38) is not enough. We have a responsibility to disseminate and discuss, both locally with airway leads of various acute care specialties, and nationally in a Canadian Airway Network that includes all disciplines. We also have a responsibility to provide cognitive aids for easy access and understanding during airway emergencies and practice them regularly. Finally, we have the responsibility to educate not just the airway managers, but also the entire team involved, by creating an algorithmic Advanced Airway Life Support (AALS) approach similar to ATLS and ACLS. Howard et al.'s findings shine a light on how the apprenticeship model fails to provide consistent, reliable, predictable airway management education. It's time we create a shared path in the forest of airway guidelines.

\section{Dans une jungle de directives sur les voies aériennes, il est temps de tracer un chemin commun}

La littérature médicale a subi une croissance exponentielle au cours des 60 dernières années, et l'anesthésiologie ne fait pas exception. ${ }^{1}$ Il est impossible de rester à jour - et 
encore moins d'intégrer la littérature principale pertinente à sa pratique, d'où la croissance de la littérature de synthèse, qui prend la forme de divers types de comptes rendus et lignes directrices de pratique clinique. Un nombre non négligeable de travaux scientifiques ont été publiés sur la façon de créer ce type de lignes directrices. ${ }^{2}$ Toutefois l'application des connaissances ${ }^{3}$ - sous la forme d'une diffusion efficace, d'une adoption et d'un changement de pratique mesurés et, plus important encore, d'une quantification de l'amélioration des devenirs centrés sur le patient - demeure souvent floue.

Les lignes directrices de pratique clinique sont publiées par divers groupes et sociétés. Destinées à aider à la prise de décision clinique, ces lignes directrices peuvent se contredire mutuellement en fonction des données probantes utilisées, des processus d'évaluation, ou encore des opinions de leurs membres. Sous peine de devenir rapidement obsolètes, les lignes directrices de pratique clinique devraient inclure un plan de révision itératif une fois publiées. Les premières lignes directrices sur la prise en charge de voies aériennes difficiles ont été publiées en 1993 par l'American Society of Anesthesiologists (ASA). ${ }^{4}$ Les premières recommandations du Canadian Airway Focus Group (CAFG) ont été publiées en $1998 .^{5}$ En 2013, les recommandations du CAFG ont été révisées et ont pris la forme de deux articles distincts, le premier portant sur les voies aériennes difficiles non anticipées, ${ }^{6}$ et le second abordant les voies aériennes difficiles anticipées. ${ }^{7}$ À ce jour, les recommandations du CAFG ont été les seules lignes directrices de pratique clinique majeures sur les voies aériennes à publier un algorithme abordant spécifiquement la prise en charge d'un patient présentant des voies aériennes difficiles anticipées. Globalement, dans une étude ciblée récente, un total de 38 algorithmes pour des voies aériennes difficiles ont été identifiés, dont $58 \%$ ont été publiés au cours des cinq dernières années (figure). ${ }^{8}$

Dans ce numéro du Journal, Howard et coll. ${ }^{9}$ rapportent les résultats d'un sondage sur l'utilisation des lignes directrices de prise en charge des voies aériennes difficiles lors de la pratique de l'anesthésiologie dans la région de London, en Ontario. Leur sondage comporte des questions précises touchant à la sensibilisation à et à l'utilisation des lignes directrices du CAFG. Dans leur sondage, les auteurs ont interrogé des anesthésiologistes et des résidents de deux centres universitaires; un sondage distinct portant sur l'adoption à l'échelle départementale des lignes directrices a été envoyé aux chefs de département de 21 services d'anesthésie communautaire de la région. Le sondage demandait aux répondants quelles lignes directrices sur les voies aériennes difficiles ils suivaient (le cas échéant), si leur département endossait une ligne directrice spécifique sur les voies aériennes difficiles, et quelle était leur connaissance des lignes directrices du CAFG. Le sondage a également exploré le niveau d'aisance personnelle des répondants avec des compétences techniques allant de techniques couramment utilisées, telles que la vidéolaryngoscopie, à d'autres compétences moins fréquentes mais non moins fondamentales nécessaires à la mise en place d'un accès aux voies aériennes d'urgence à l'avant du cou (cricothyroïdotomie), généralement lors d'une urgence « impossible de ventiler, impossible d'oxygéner ».

Les taux de réponse au sondage dans les deux centres universitaires étaient raisonnables : $74 \%$ (28/38) des résidents et $68 \%(51 / 74)$ des anesthésiologistes universitaires y ont participé. Cependant, la réponse des chefs de département régionaux a été quelque peu décevante, avec seulement $52 \%$ (11/21) choisissant de participer; par conséquent, les modèles ou les conclusions glanés des départements régionaux d'anesthésie sont limités.

Une minorité notable de $25 \%$ des anesthésiologistes universitaires (13/51) et un petit nombre de résidents (7\% [2/28]) ont répondu qu'ils ne suivaient aucune ligne directrice publiée sur les voies aériennes. Parmi les anesthésiologistes n'utilisant pas de lignes directrices, la raison la plus souvent invoquée était l'opinion selon laquelle la prise en charge des voies aériennes ne devrait pas être standardisée. Cet avis est fondé; chaque patient mérite une stratégie de prise en charge des voies aériennes (c.-à-d., une série coordonnée de plans) basée sur ses caractéristiques et circonstances individuelles. L'ironie est que les lignes directrices sur les voies aériennes n'épousent pas l'idée « d'une stratégie de voies aériennes pour tous »; plutôt, elles mettent en évidence des facteurs importants ( $\mathrm{p}$. ex. disponibilité du personnel et de l'équipement, physiologie du patient) à prendre en compte lors de l'élaboration d'une stratégie individuelle pour la prise en charge des voies aériennes.

Trois domaines principaux composent le développement d'une expertise pour la prise en charge des voies aériennes : la prise de décision appropriée en fonction d'un contexte spécifique, les compétences techniques, et un travail d'équipe prévisible. Bien que des lignes directrices puissent aider à la prise de décision, les compétences techniques ont traditionnellement été acquises grâce à un modèle par apprentissage. ${ }^{10}$ Le modèle par apprentissage est basé sur l'hypothèse qu'avec suffisamment de temps, l'apprenant aura été adéquatement exposé à tous les aspects de la prise de décision et aux compétences techniques en matière de prise en charge des voies aériennes, grâce au simple volume de patients dont il ou elle aura pris soin. Bien que cela puisse suffire à obtenir une formation appropriée en ce qui touche aux compétences plus couramment utilisées (p. ex., Howard et coll. ont constaté que $90 \%$ des répondants se sentaient à l'aise avec la 
vidéolaryngoscopie), un tel niveau d'aisance ne se traduit pas forcément dans la prise de décision en cas d'urgence ${ }^{11}$ ou dans l'exécution de gestes rares (par exemple, une cricothyroïdotomie). Le modèle d'apprentissage endosse également un agenda caché, c'est-à-dire que la prise en charge des voies aériennes est une initiative individuelle et non un effort d'équipe.

Parmi les lignes directrices suivies par les répondants dans le sondage de Howard et coll. figuraient celles de l'ASA, du CAFG et de la Difficult Airway Society. ${ }^{12}$ Même si $72 \%$ des répondants connaissaient les lignes directrices du CAFG, bon nombre d'entre eux ont mentionné des problèmes liés à l'application des connaissances. Les critiques incluaient l'absence d'endossement de ces lignes directrices par une société professionnelle, le manque de diffusion adéquate, et le manque d'aides cognitives associées pour aider le responsable des voies aériennes pendant les soins aux patients. Toutes ces critiques sont justifiées. Il n'est pas acceptable de publier des lignes directrices de pratique clinique et de laisser aux cliniciens le soin de les trouver et de les rendre applicables. Les lignes directrices de pratique clinique ont l'obligation de se rendre conviviales et adaptables à la pratique locale tout en incluant des aides cognitives / aides-mémoire afin d'encourager leur utilisation. Bien que l'endossement par une société professionnelle soit traditionnel, la prise en charge des voies aériennes est pratiquée par plusieurs spécialités de soins aigus (p. ex. médecine d'urgence, soins intensifs, anesthésiologie) et d'autres domaines des soins de santé (p. ex. inhalothérapie, soins paramédicaux préhospitaliers). Il est temps de créer un Réseau canadien des voies aériennes pour mettre en relation les responsables des voies aériennes de toutes les spécialités, et pas seulement par le biais de lignes directrices intermittentes du CAFG. Un tel réseau est en cours de développement. Des responsables départementaux des voies aériennes, un concept issu du $4^{\mathrm{e}}$ projet d'audit national du RoyaumeUni, ${ }^{13}$ constituent un moyen d'associer un ensemble de lignes directrices de pratique à une personne-ressource clairement identifiée au niveau local. Au Royaume-Uni et en Nouvelle-Zélande, il existe aujourd'hui des responsables des voies aériennes dont la tâche est de traduire les connaissances ${ }^{14}$; ils constituent un moyen de rendre les lignes directrices itératives et sensibles aux exigences locales.

Il faut souligner que, dans le sondage de Howard et coll., $25 \%$ des résidents ont déclaré avoir appris et suivre plusieurs lignes directrices. Bien que cela ne soit pas nécessairement mal en soi, cette situation met également en évidence un paradoxe: la moitié des résidents ont également rapporté avoir eu une formation inadéquate en matière de prise en charge des voies aériennes difficiles. En ce sens, l'étude de Howard et coll. met le doigt sur la faiblesse du système par apprentissage : il peut surenseigner certains aspects de la prise en charge des voies aériennes tout en en sous-enseignant d'autres. Tous les résidents méritent de pouvoir suivre un programme d'études délibéré et bien conçu sur les voies aériennes, qui soit à la fois cohérent, fondé sur des données probantes, et complet. Un tel programme doit inclure les compétences décisionnelles, techniques et de travail d'équipe requises pour la prévention et la prise en charge des urgences des voies aériennes. Si nous ne délimitons pas, n'enseignons pas et n'évaluons pas clairement ces compétences, nous rendons un mauvais service à nos résidents et à nos patients.

Enfin, aucune ligne directrice sur les voies aériennes difficiles ne serait complète si elle n'abordait pas spécifiquement l'urgence «impossible de ventiler, impossible d'oxygéner ». Même s'il existe de nombreuses lignes directrices sur les voies aériennes difficiles, ${ }^{8}$ l'approche d'une urgence «impossible de ventiler, impossible d'oxygéner» est relativement uniforme. La réalisation d'une cricothyroïdotomie constitue une compétence fondamentale des compétences avancées en matière de voies aériennes. Tous les intervenants dans la prise en charge avancée des voies aériennes ont la responsabilité de savoir quand et comment réaliser une cricothyrö̈dotomie d'urgence - sans chirurgien. Howard et coll. ont constaté que seule une minorité de résidents (4 à $7 \%$ ) et d'anesthésiologistes (18 à $24 \%$ ) se sentaient à l'aise de réaliser une cricothyroïdotomie avec une aiguille ou un scalpel. Cela se reflète dans les réclamations pour faute professionnelle liées aux intubations trachéales difficiles dans la base de données du Projet de réclamations réglées de l'ASA, où le délai avant une cricothyroïdotomie d'urgence, en général pour cause d'attente d'un chirurgien, a été cité comme un thème commun dans la majorité des cas signalés. ${ }^{15} \mathrm{Il}$ existe aujourd'hui des ressources facilement accessibles pour pratiquer la cricothyroïdotomie. ${ }^{16}$ Peut-être que le fait qu'Howard et coll. aient remarqué que seulement $13 \%$ des répondants avaient réalisé une cricothyroïdotomie d'urgence au cours de la dernière année et que $43 \%$ n'en avaient jamais réalisée peut maintenant être abordé.

Comment pouvons-nous faire mieux? La prise en charge appropriée d'une situation «impossible de ventiler, impossible d'oxygèner» ne devrait pas dépendre uniquement de la conscience situationnelle, de la prise de décision, et des compétences cognitives et techniques d'un seul individu responsable de la prise en charge des voies aériennes qui les exécuterait avec précision et en temps opportun. Cela n'est pas réaliste et pourrait être potentiellement destructeur de sa carrière pour le responsable des voies aériennes, inutilement risqué pour le patient et dommageable pour l'équipe, cette dernière 
comptant souvent des personnes ayant une meilleure conscience situationnelle que le responsable des voies aériennes. L'analyse de la base de données du Projet de réclamations réglées liées à une intubation trachéale difficile de l'ASA a révélé que la persistance des tentatives d'intubation trachéale constituait un problème récurrent ${ }^{15}$; cela remet en question la notion d' $\mathrm{d}^{\text {impossible }}$ d'intuber », surtout si le responsable des voies aériennes a la responsabilité de déterminer cette situation. La publication récente du Journal sur des cas juridiques civils réglés liés à la prise en charge des voies aériennes par des anesthésiologistes spécialisés provenant de l'Association canadienne de protection médicale a révélé que la majorité des patients qui avaient subi des lésions cérébrales ou qui étaient décédés étaient des patients de statut physique ASA I à III se présentant pour une chirurgie non urgente. ${ }^{17}$ Les situations "impossible de ventiler, impossible d'oxygéner » peuvent survenir à tout moment; la différence entre la vie et la mort d'un patient reposera souvent sur le fait de disposer de réponses prévisibles aux urgences en matière de voies aériennes dans les limites locales de l'équipement, des compétences et de la pratique.

Les soins de traumatologie aiguë sont gérés à l'aide d'une approche standardisée de Soins avancés de réanimation des patients polytraumatisés (ATLS Advanced Trauma Life Support), de sorte qu'aucune lésion grave n'est manquée et que plusieurs fournisseurs de soins de santé peuvent effectuer des tâches simultanément avec un responsable (leader). Les détails des lésions peuvent alors être pris en charge. Les conditions cardiaques aiguës sont prises en charge à l'aide d'une approche standardisée de Soins avancés en réanimation cardiovasculaire (SARC ou ACLS en anglais) pour rétablir la circulation. Les compressions thoraciques sont anticipées et effectuées par un groupe de personnes en rotation pendant que des médicaments standards sont administrés. Les détails spécifiques de la prise en charge du rythme peuvent alors être au centre de l'attention. Le responsable est au pied du lit. Si l'équipe ne partage pas les tâches et ne prépare pas l'équipement pour les prochaines étapes prévues, les soins sont retardés. Il est temps de disposer d'une telle approche standardisée des urgences respiratoires. Bien qu'il existe beaucoup de lignes directrices portant sur les voies aériennes difficiles, une situation « impossible de ventiler, impossible d'oxygéner » peut être identifiée par l'équipe par la capnographie sur écran et la désaturation oximétrique, habituellement dans cet ordre. Cela ne dépend pas du responsable des voies aériennes déterminant l'événement qui se produit. Dans une approche de prise en charge avancée des voies aériennes, un dispositif supraglottique serait alors immédiatement passé au responsable des voies aériennes, tandis qu'un code voies aériennes serait signalé. Une cricothyroïdotomie d'urgence est à prévoir si la ventilation au masque, l'utilisation d'un dispositif supraglottique et l'intubation trachéale par vidéolaryngoscopie et laryngoscopie directe ne parviennent pas à oxygéner le patient. La fréquence d'exercices de pratique pour un tel événement pourrait être similaire à la recertification pour les autres soins d'urgence. Bien qu'il s'agisse d'une méthode imparfaite, le fait de fournir un cadre de réponses standard libère de l'espace cognitif pour résoudre les problèmes spécifiques à la situation spécifique, tout comme le font les ATLS et SARC. La pratique rend l'inconnu familier, et les urgences de type « impossible de ventiler, impossible d'oxygéner », tout comme la réalisation d'une cricothyroïdotomie d'urgence, ne font pas exception à la règle.

En conclusion, Howard et coll. nous ont fourni une perspective cruciale : la production de lignes directrices de pratique clinique (qu'il s'agisse d'une ligne directrice ou de 38) pour la prise en charge des voies aériennes difficiles ne suffit pas. Nous avons la responsabilité de les diffuser et de les discuter, à la fois à l'échelle locale, avec les responsables des voies aériennes de diverses spécialités de soins aigus, et à l'échelle nationale, dans un Réseau canadien des voies aériennes qui englobe toutes les disciplines. Nous avons également la responsabilité de proposer des aides cognitives facilement accessibles et compréhensibles lors d'urgences des voies aériennes et de s'exercer à les utiliser régulièrement. Enfin, nous avons la responsabilité d'éduquer non seulement les responsables des voies aériennes, mais aussi toute l'équipe impliquée, en créant une approche fondée sur un algorithme de Soins avancés en réanimation respiratoire (SARR) similaire aux ATLS et SARC. Les conclusions de Howard et coll. mettent en lumière la façon dont le modèle d'apprentissage ne parvient pas à fournir une formation cohérente, fiable et prévisible en matière de prise en charge des voies aériennes. Il est temps de tracer un chemin commun dans la jungle de directives sur les voies aériennes.

\section{Disclosures None.}

Funding statement None.

Editorial responsibility This submission was handled by Dr. Stephan K.W. Schwarz, Editor-in-Chief, Canadian Journal of Anesthesia.

Déclaration Aucune.

Déclaration de financement Aucune.

Responsabilité éditoriale Ce manuscrit a été traité par Dr Stephan K.W. Schwarz, rédacteur en chef, Journal canadien d'anesthésie. 


\section{References}

1. Anonymous. The growth of medical literature. JAMA 2013; DOI: 10.1001/jama.2013.5484.

2. Brouwers $M C$, Kho ME, Browman GP, et al. AGREE II: advancing guideline development, reporting and evaluation in health care. CMAJ 2010; 182: E839-42.

3. Canadian Institutes of Health Research. Knowledge Translation. Available from URL: https://cihr-irsc.gc.ca/e/29418.html (accessed May 2021).

4. Anonymous. Practice guidelines for management of the difficult airway. A report by the American Society of Anesthesiologists Task Force on Management of the Difficult Airway. Anesthesiology 1993; 78: 597-602.

5. Crosby ET, Cooper RM, Douglas MJ, et al. The unanticipated difficult airway with recommendations for management. Can $\mathbf{J}$ Anaesth 1998; 45: 757-76.

6. Law JA, Broemling N, Cooper RM, et al. The difficult airway with recommendations for management - part 1 - difficult tracheal intubation encountered in an unconscious/induced patient. Can J Anesth 2013; 60: 1089-118.

7. Law JA, Broemling N, Cooper RM, et al. The difficult airway with recommendations for management - part 2 - the anticipated difficult airway. Can J Anesth 2013; 60: 1119-38.

8. Edelman DA, Perkins EJ, Brewster DJ. Difficult airway management algorithms: a directed review. Anaesthesia 2019; 74: $1175-85$.

9. Howard M, Noppens R, Gonzalez N, Jones P, Payne SM. Seven years on from the Canadian Airway Focus Group Difficult Airway Guidelines: an observational survey. Can J Anesth 2021; this issue.

10. Marshall SD, Chrimes $N$. Time for a breath of fresh air: rethinking training in airway management. Anaesthesia 2016; 71 : 1259-64.
11. Thomsen JL, Nфrskov AK, Rosenstock CV. Supraglottic airway devices in difficult airway management: a retrospective cohort study of 658,104 general anaesthetics registered in the Danish Anaesthesia Database. Anaesthesia 2019; 74: 151-7.

12. Frerk C, Mitchell VS, McNarry AF, et al. Difficult Airway Society 2015 guidelines for management of unanticipated difficult intubation in adults. Br J Anaesth 2015; 115: 827-48.

13. Cook T, Woodall N, Frerk C; The Royal College of Anaesthetists; The Difficult Airway Society. NAP4 - 4th National Audit Project of the Royal College of Anaesthetists and the Difficult Airway Society Major complications of airway management in the United Kingdom: report and findings. March 2011. Available from URL: https://www.nationalauditprojects.org.uk/downloads/ NAP4\%20Full\%20Report.pdf (accessed May 2021).

14. McNarry AF, Cook TM, Baker PA, O'Sullivan EP. The Airway Lead: opportunities to improve institutional and personal preparedness for airway management. Br J Anaesth 2020; 125: e22-4.

15. Joffe AM, Aziz MF, Posner KL, Duggan LV, Mincer SL, Domino $K B$. Management of difficult tracheal intubation: a closed claim analysis. Anesthesiology 2019; 131: 818-29.

16. Duggan LV, Lockhart SL, Cook TM, O'Sullivan EP, Date T, Baker PA. The Airway App: exploring the role of smartphone technology to capture emergency front-of-neck airway experiences internationally. Anaesthesia 2018; 73: 703-10.

17. Crosby ET, Duggan LV, Finestone PJ, Liu R, De Gorter R, Calder LA. Anesthesiology airway-related medicolegal cases from the Canadian Medical Protection Association. Can J Anesth 2021; 68: 183-95.

Publisher's Note Springer Nature remains neutral with regard to jurisdictional claims in published maps and institutional affiliations. 